\title{
Equações Lineares no Ensino Médio: uma proposta didática por meio da Resolução de Problemas
}

\author{
Linear Equations in High School: a didactic proposal through Problem Solving
}

\begin{abstract}
Glaucia Maria Bressan Universidade Tecnológica Federal do Paraná (UTFPR), Departamento Acadêmico de Matemática Campus Cornélio Procópio, Cornélio Procópio, PR, Brasil http://orcid.org/0000-0001-6996-3129, glauciabressan@utfpr.edu.br
\end{abstract}

\author{
Elenice Weber Stiegelmeier \\ Universidade Tecnológica Federal do Paraná (UTFPR), Departamento Acadêmico de Matemática \\ Campus Cornélio Procópio, Cornélio Procópio, PR, Brasil \\ http://orcid.org/0000-0002-8834-4937, elenicew@utfpr.edu.br
}

\begin{tabular}{l}
\hline Informações do Artigo \\
\hline cc) (†) \$ \\
\hline $\mathrm{NY}$
\end{tabular}

Histórico do Artigo

Submissão: 30 de janeiro de 2019.

Aceite: 25 de abril de 2019 .

Palavras-chave

Resolução de Problemas

Ensino de Equações Lineares

Otimização Linear

\section{Resumo}

Nesse artigo apresentamos uma abordagem do ensino de Equações Lineares por meio da Resolução de Problemas, desde suas contribuições teóricas para o estudo de funções lineares à sua aplicação em um grupo de estudantes do primeiro ano do Ensino Médio, os quais faziam parte do Programa de Iniciação Científica para o Ensino Médio (PIBIC-EM), promovido pelo CNPq e executado na Universidade Tecnológica Federal do Paraná, Campus Cornélio Procópio. As atividades desenvolvidas foram voltadas ao ensino de Funções Lineares por meio da Resolução de Problemas e promoveram a integração dos alunos do Ensino Médio com a universidade, estimulando o raciocínio matemático, a capacidade de resolver problemas e a socialização. Os resultados indicam que o uso da Resolução de Problemas como metodologia de ensino potencializa a construção do saber matemático aos alunos do Ensino Médio. Ademais, concluímos que o uso da Resolução de Problemas se apresenta como uma possibilidade de se imprimir maior significado à construção dos conceitos matemáticos, uma vez que a formalização dos conceitos e teorias matemáticas, feita pelo professor, passa a ter mais significado para os estudantes.

\section{Abstract}

Keywords

Problems Solving

Linear Equation Teaching

Linear Optimization

\begin{abstract}
In this paper we present an approach of the teaching of Linear Equations through Problems Solving, from his theoretical contributions to the study of linear functions to his application in a group of students of the first year of High School, who was part of the Scientific Initiation Program for Higher Education (PIBIC-EM), promoted by CNPq and executed in the Universidade Tecnológica Federal do Paraná, Campus Cornélio Procópio. The developed activities were developed involved the teaching of Linear Functions unsing Problem Solving and promoted the integration of high school students with the university, stimulating mathematical reasoning, problems solving and socialization. The results indicate that the use of Problems Solving as teaching methodology enhances the construction of mathematical knowledge for High School students. In addition, we conclude that the use of Problem Solving is a possibility to give greater meaning to the construction of mathematical concepts, since the formalization of concepts and mathematical theories, made by the teacher, becomes more meaningful for students.
\end{abstract}




\section{Introdução}

O ensino tem estado em constante mudança, especialmente nas últimas décadas, devido ao acesso às diversas formas de tecnologias. Assim, o professor deve buscar se reinventar e implementar novas metodologias, que estejam mais próximas do universo dos alunos (FONSECA, 2005). Levando em consideração o exposto acima e atentando-se ao fato de que os alunos estão inseridos em um cenário no qual a tecnologia tem evoluído numa proporção muito rápida, nem sempre acompanhada pelo processo de ensino-aprendizagem, novas formas de ensinar devem ser discutidas nessa fase de mudanças, a fim de estimular a prática docente por meio de novos instrumentos tecnológicos que visem auxiliar o professor no processo de ensino-aprendizagem.

Além disso, é importante colocar o discente frente a situações reais, que relacionem a matemática a problemas práticos. Isso faz com que o aluno se sinta desafiado e, com isso, o professor pode motivá-lo à busca pelo raciocínio independente, criando um ambiente em que o aluno passa a ser o autor de seus conhecimentos, e o professor, o mediador deste processo, auxiliando os alunos por meio de indagações estimulantes e incutindo-lhes o gosto pela matemática. Nesse âmbito, um dos deveres do professor é o de investigar e o de desafiar os seus alunos (POLYA, 2006).

Nessa perspectiva, a Resolução de Problemas proposta pela autora contribui para o estudo em questão, uma vez que levar para a sala de aula problemas do cotidiano contribui para despertar a curiosidade dos alunos por novos conhecimentos, a fim de solucionar o problema apresentado.

Neste sentido, um dos ramos da matemática aplicada que se ocupa em resolver problemas em situações reais é a Pesquisa Operacional, que pode ser definida como uma ciência que busca modelar matematicamente problemas quantitativos, agregando métodos matemáticos e estatísticos que auxiliam na tomada de decisões (GOLDBARG; LUNA, 2005; LACHTERMACHER, 2009). E, ainda, a Pesquisa Operacional se destaca por congregar diversas das mais consagradas técnicas de modelagem matemática (GOLDBARG; LUNA, 2005; LINS; CALOBA, 2006; LACHTERMACHER, 2009).

A Otimização Linear, por sua vez, é uma das mais importantes variedades dos modelos quantitativos que apresenta uma grande utilidade na solução (exata) de problemas de otimização (GOLDBARG; LUNA, 2005). Consiste de uma área da Pesquisa Operacional em que os algoritmos e as técnicas buscam estruturar e solucionar modelos quantitativos que podem ser expressos matematicamente por meio de equações lineares. Desta forma, a Otimização Linear é uma das áreas de aplicações da Matemática que auxilia na resolução de problemas relacionados à administração, engenharias, logística e transporte, dentre outros, e que pode ser explorada, em um nível mais elementar, no Ensino Médio, para o ensino de equações lineares e seus gráficos através do uso de situações reais que contribuem para a construção do conhecimento.

Os modelos matemáticos envolvendo problemas de otimização são estruturados logicamente com o objetivo de determinar as melhores condições de funcionamento para os 
sistemas representados. Desta forma, alguns métodos algébricos podem ser apresentados de forma acessível ao estudante do Ensino Médio, para resolução de problemas de otimização que envolvem duas variáveis. Como a Matemática também deve ser vista como um conhecimento para desenvolver o raciocínio, aliar otimização à Resolução de Problemas pode ser um método eficiente para o estímulo ao desenvolvimento lógico.

$\mathrm{Na}$ literatura, alguns trabalhos abordam esse tema. Rocha (2013) apresenta alguns métodos que podem ser trabalhados com estudantes do Ensino Médio, para resolução de problemas simples de otimização. Dentre estes, destacam-se a otimização de funções quadráticas, funções discretas, algumas funções contínuas, além de aplicações da desigualdade das médias. No trabalho de Azevedo (2015), procurou-se mostrar algumas aplicações matemáticas simples e detalhadas, por meio de certos problemas de otimização em nível de Ensino Médio. E, também, é apresentada uma breve história do Cálculo e uma síntese de resultados cujo desenvolvimento foi realizado com base em funções reais trabalhadas no Ensino Médio.

Portanto, o objetivo do presente trabalho é investigar uma proposta sobre o ensino de equações lineares e seus gráficos por meio da Resolução de Problemas como metodologia de ensino. A metodologia desta pesquisa está baseada nos princípios da Resolução de Problemas apresentada por Polya (2006) uma vez que através da resolução de problemas o aluno se torna capaz de compreender o papel da matemática no seu cotidiano.

\section{Referencial teórico}

\subsection{Resolução de Problemas}

A Resolução de Problemas vem ganhando espaço nos currículos escolares diante da necessidade de se buscar novas metodologias de ensino que valorizem a construção do saber, diferentemente da metodologia tradicional, a qual está baseada na formalidade matemática e no rigor dos conceitos.

Leal Junior e Onuchic (2015) justificam o uso da Resolução de problemas como uma metodologia para ensinar matemática baseados no fato de que o problema "[...] inicia e fomenta a construção e a formação de um novo conceito matemático, por meio de sua produção ativa e da constituição da Matemática através da sua prática" (p. 958-959). Logo, os estudantes passam a ter um papel ativo na construção dos saberes, e os professores são os mediadores desse processo.

Nessa perspectiva, a Resolução de Problemas passa a ser uma atividade importante para os alunos uma vez que "a criatividade atua como uma potência inerente aos agenciamentos e aos acontecimentos no pensamento dos estudantes, que ao se depararem com os problemas, buscarão meios para resolvê-los" (LEAL JUNIOR; ONUCHIC, 2015, p. 960). E, ainda, outro ponto que os autores destacam nesse processo é a "invenção, a qual [...] tem seu início na concepção do problema, onde o professor o inventa com base no construto sócio-histórico-cultural para estimular 
a aprendizagem dos estudantes e subverter o modelo representacionalista e tradicional de ensino" (LEAL JUNIOR; ONUCHIC, 2015, p. 960).

Nesse processo, o professor tem um papel importante a desempenhar, pois deve estimular seus alunos em sala de aula, proporcionar formas diferenciadas de conhecimento tornando os alunos mais independentes. Portanto, o professor passa a ser um mediador do conhecimento matemático tornando-se uma peça chave no ensino-aprendizagem (SANTOS; ALMEIDA, 2018).

Para Polya (2006), a metodologia da Resolução de Problemas busca dividir a solução de um problema em quatro fases: compreensão do problema, estabelecimento de um plano, execução do plano e um retrospecto da resolução completa, revendo-a e discutindo-a. Cada uma destas fases tem importância no desenvolvimento do raciocínio lógico do aluno para a resolução de problemas.

Para a aplicabilidade da metodologia de ensino através da Resolução de Problemas, Onuchic et al. (2014) criaram um roteiro para auxiliar os professores na elaboração do planejamento de suas aulas, contendo uma sequência de atividades que devem ser desenvolvidas, a fim de fornecer subsídios para a atuação de professor e dos estudantes nesse processo. São eles (ONUCHIC et al., 2014 apud SILVA; GUERRA, 2017, p. 43):

1. Proposição do problema - Seleciona ou elabora um problema e denomina-se de problema gerador.

2. Leitura individual - Distribuir uma cópia impressa do problema para cada aluno e solicitar a leitura do mesmo.

3. Leitura em conjunto - Distribuir a turma em pequenos grupos e, solicitar uma nova leitura do problema.

4. Resolução do problema - A partir do momento em que o aluno entendeu o problema tenta resolvê-lo, em grupo, permitindo assim a construção do conhecimento sobre o conteúdo que o professor planejou para aquela aula.

5. Observar e incentivar - Nesse momento, o professor muda de comunicador do conhecimento para o de observador, organizador, consultor, mediador, interventor, controlador, incentivador da aprendizagem.

6. Registro das resoluções na lousa - Anotar os resultados obtidos pelos grupos quer sejam certo ou errado e aqueles feitos por diferentes caminhos.

7. Plenária - Assembleia com todos os alunos. Como todos trabalham sobre o problema dado, estão ansiosos quanto a seus resultados, dessa forma, participam.

8. Busca do consenso - Após as discussões e sanadas as dúvidas, o professor juntamente com os alunos tentam chegar a um consenso.

9. Formalização do conteúdo - Faz-se uma síntese daquilo que se objetivava "aprender" a partir do problema gerador. São colocadas as devidas definições, identificando propriedades, fazendo demonstrações, etc.

10. Proposição e resolução de novos problemas - Nesta etapa, após a formalização do conteúdo, propõem-se novos problemas para a fixação de aprendizagem.

Leal Junior e Onuchic (2015), também, destacam a necessidade do professor estar livre das amarras de conteúdos e currículos durante este processo, pois podem surgir novos problemas que 
levem a caminhos diferentes dos planejados, mas não menos importantes na construção do conhecimento.

\subsection{Conceitos básicos de otimização linear para o Ensino Médio}

$\mathrm{Na}$ formulação geral de um problema de otimização linear, se existem $n$ decisões a serem tomadas é associada uma variável a cada um dos valores quantitativos do problema e esta variável é chamada de variável de decisão. Ao se aplicar um método de resolução, o valor dessas variáveis é determinado e a solução ótima é encontrada, ou seja, a melhor solução, dentre todas as possíveis.

O objetivo do problema é aquilo que se pretende minimizar ou maximizar. Logo, uma função numérica das variáveis de decisão, chamada de função objetivo, é então estruturada. Deve-se também analisar quais são as limitações impostas ao problema, que devem ser expressas matematicamente por meio de equações e/ou inequações lineares, chamadas de restrições do problema. Inerente aos problemas de Otimização Linear, está a condição de que todas as variáveis de decisão pertencem ao primeiro quadrante, ou seja, são maiores ou iguais a zero (GOLDBARG; LUNA, 2005; LINS; CALOBA, 2006; LACHTERMACHER, 2009).

A partir da modelagem matemática de um problema descrito como um problema de Otimização Linear, pode-se encontrar a sua solução por meio da interpretação gráfica da função objetivo, isto é, o que se pretende minimizar ou maximizar, e das restrições operacionais, desde que o problema possua duas variáveis de decisão. Algumas definições fundamentais da Otimização Linear são descritas a seguir (LINS; CALOBA, 2006):

- Região Viável: conjunto de soluções que satisfazem as restrições do problema.

- Solução Viável: solução que pertence à região viável.

- Vértices: pontos de interseção das restrições do problema.

- Vértices da Região Viável: pontos de interseção das restrições do problema que fazem parte da região viável.

Após a formulação matemática do problema com um problema de programação linear, os passos para obter a solução ótima são os seguintes:

- Considerar as restrições do problema como igualdades e traçar as retas correspondentes.

- Determinar a região viável, a partir das desigualdades das restrições (polígono convexo).

- Identificar os vértices da região viável como pontos $\left(x_{1}, x_{2}\right)$.

- Substituir os valores dos pontos dos vértices na função objetivo e verificar qual deles fornece o maior valor (se o problema for de maximização) ou o menor valor (se o problema for de minimização). 
Durante a construção das retas no plano cartesiano, os alunos podem se deparar com duas situações:

$1^{\circ}$. Problemas com múltiplas soluções: ocorrem quando mais de um vértice apresenta a mesma solução ótima, indicando sobreposição de retas que representam as restrições. Todos os pontos são solução ótima (LINS; CALOBA, 2006).

$2^{\circ}$. Problemas com solução ilimitada: ocorrem quando a região viável é aberta, ou seja, a solução melhora conforme a expansão da região e não há uma restrição que a limite (LINS; CALOBA, 2006).

Desta forma, em um ambiente de Ensino Médio, os professores podem propor atividades e desafios para que os alunos possam discutir as possíveis soluções e os caminhos para encontrálas. Assim, a metodologia de ensino da Resolução de Problema se mostra como uma ferramenta adequada para melhorar a relação entre os alunos e o conteúdo matemático.

\section{Metodologia}

Conforme Schoroeder e Lester (1989, apud ONUCHIC, 1999, p. 206), há três maneiras de abordar a Resolução de Problemas: ensinar sobre resolução de problemas, ensinar a resolução de problemas e ensinar matemática por meio da resolução de problemas. O professor que ressalta a metodologia de resolução de problemas de Polya ou alguma variação dele busca ensinar sobre resolução de problemas.

Para o desenvolvimento do trabalho, a metodologia de ensino da Resolução de Problemas foi baseada em Polya (2006, p. 4), o qual aborda a resolução de problemas em quatro fases:

Primeiro, temos de compreender o problema, temos de perceber claramente o que é necessário. Segundo, temos de ver como os diversos itens estão interrelacionados, como a incógnita está ligada aos dados, para termos a ideia da resolução, para estabelecermos um plano. Terceiro, executamos o nosso plano. Quarto, fazemos um retrospecto da resolução completa, revendo-a e discutindo-a.

A pesquisa foi desenvolvida com alunos do $1^{\circ}$ ano do Ensino Médio da Escola Estadual Monteiro Lobato localizada em Cornélio Procópio, os quais faziam parte do grupo de alunos participantes do PIBIC-EM desenvolvido na UTFPR-CP.

Com o propósito de identificar os saberes prévios que os alunos possuíam acerca do tema, foram realizados dois encontros com o objetivo de rever os conteúdos básicos necessários para o desenvolvimento das atividades.

No terceiro encontro foram aplicadas as atividades referentes à proposta didática usando como estratégia de ensino a Resolução de Problemas com base nas quatro fases definidas por Polya (2006). Na primeira fase, os alunos têm contato inicial com o problema, em que o objetivo foi despertar no aluno a curiosidade, a busca por um padrão matemático e a troca de ideias entre os alunos, ou seja, nesse momento o aluno deve compreender o problema. Na segunda fase, os alunos 
devem definir um plano e, para isso, definir as incógnitas do problema e como estas estão relacionadas. Na terceira fase, os alunos executam o plano definido na etapa anterior, propondo conjecturas e formas de resolução, com base no conhecimento sobre o tema. E, finalmente, os alunos devem fazer a verificação da solução encontrada e discutir sobre a resolução do problema.

Para a execução das atividades foram propostos dois problemas de otimização linear com duas variáveis de decisão. Os conteúdos abordados referiam-se às equações lineares, gráficos de retas no plano cartesiano e interseção de retas. Nos Quadros 1 e 2 são apresentados os problemas geradores abordados com os alunos. O problema gerador é considerado como aquele que visa a construção de um novo conceito, conteúdo ou procedimento matemático (ONUCHIC, 2013).

Quadro 1 - Problema gerador referente ao conteúdo de equações lineares e seus gráficos. Problema 1 (Plantio de soja e algodão)

Para uma determinada área, utilizada para o plantio de soja e algodão, calcula-se que há 800 homens-horas disponíveis durante o período de semeadura, e que são necessários 20 homens-horas por hectare de soja e 40 homens-horas por hectare de algodão. Oferece-se ainda uma linha máxima de crédito de $\$ 6.000,00$, dividida da seguinte forma: $\$ 300,00$ por hectare de soja e $\$ 100,00$ por hectare de algodão. Como organizar esta área de plantio se é sabido que as margens de lucro esperadas são $\$ 100,00$ por hectare de soja e $\$ 80,00$ por hectare de algodão.

Fonte: Dados da pesquisa.

Quadro 2 - Problema gerador referente a tomada de decisão em um problema de otimização linear.

Problema 2 (Tomada de decisão - Oferta de emprego)

Consiste em escolher qual a melhor opção entre 2 alternativas de ofertas de emprego:

- 10 reais por hora trabalhada mais $2 \%$ de comissão sobre as vendas.

- 8 reais por hora trabalhada mais $3 \%$ de comissão sobre as vendas.

Fonte: Dados da pesquisa.

A seguir apresentamos o planejamento para a execução de cada uma das atividades referente aos problemas geradores.

- Leitura e resolução do problema gerador pelo grupo;

- Socialização dos registros do grupo no quadro;

- Discussão acerca das respostas obtidas;

- Compreensão da resposta definida para o problema gerador;

- Apresentação formal do conceito de equações lineares e seus gráficos;

- Apresentação formal do conceito de interseção de retas;

- Apresentação formal dos conceitos de otimização linear para duas variáveis.

- Proposta e resolução de novo problema gerador. 


\section{Resultados e discussão}

A execução do plano em cada problema depende da estratégia formulada pelos alunos em sala. Mostramos aqui um dos caminhos apresentados pelos alunos a fim de auxiliar o professor a trabalhar com a Otimização Linear aliada à Resolução de Problemas.

Primeiramente, foram expostos os problemas aos alunos e, em grupo, os alunos discutiram cada um dos problemas e fizerem as primeiras anotações, sem a interferência do professor. $\mathrm{Na}$ sequência, o professor fez sua primeira intervenção com o objetivo de questionar os alunos acerca do Problema Gerador 1.

Alguns dos questionamentos realizados pelo professor foram: Quais são as incógnitas do problema? Qual a notação adequada? Qual a relação das variáveis envolvidas? Qual o objetivo do problema? Onde queremos chegar?

Em um segundo momento, o professor deixou os alunos livres para criarem as conjecturas e modelos matemáticos adequados para a compreensão do problema e, por fim, chegar ao modelo matemáticos em questão. Nessa fase, os alunos buscaram estipular um plano para a resolução do problema.

A seguir, é apresentado o modelo matemático e sua resolução referente ao Problema Gerador 1, da forma como foi trabalhado com os alunos. Novamente o enunciado do problema é exposto a fim de facilitar a compreensão.

i) Problema 1 (Plantio de soja e algodão): Para uma determinada área, utilizada para o plantio de soja e algodão, calcula-se que há 800 homens-horas disponíveis durante o período de semeadura, e que são necessários 20 homens-horas por hectare de soja e 40 homens-horas por hectare de algodão. Oferece-se ainda uma linha máxima de crédito de $\$ 6.000,00$, dividida da seguinte forma: $\$ 300,00$ por hectare de soja e $\$ 100,00$ por hectare de algodão. Como organizar esta área de plantio se é sabido que as margens de lucro esperadas são $\$ 100,00$ por hectare de soja e $\$ 80,00$ por hectare de algodão.

\section{a) Estabelecendo um plano}

Inicialmente escrevemos $x_{1}$ a incógnita referente à cultura de algodão e $x_{2}$ a incógnita referente à cultura de soja.

O objetivo do problema é obter o lucro máximo representado por $Z$; logo, a representação matemática do modelo pode ser dada por:

$$
\operatorname{Max} Z=100 x_{1}+80 x_{2}
$$

Para a obtenção do lucro algumas restrições são impostas ao problema, chamamos de conjunto de restrições do problema, dadas por:

$$
\begin{gathered}
2 x_{1}+4 x_{2} \leq 80 \\
3 x_{1}+x_{2} \leq 60
\end{gathered}
$$


Outra observação do problema é que as variáveis são sempre não negativas, chamamos de restrição de não negatividade, dada por:

$$
x_{1}, x_{2} \geq 0
$$

\section{b) Execução do plano}

Para a execução do plano os alunos listaram alguns pontos pertencentes a cada uma das restrições e descreverão a relação dessas no plano cartesiano, inicialmente através da construção de tabelas e depois a construção do gráfico no plano cartesiano.

A seguir, o conjunto de alguns pontos para a restrição 1:

\begin{tabular}{c|c}
$x_{1}$ & $x_{2}$ \\
\hline 0 & 20 \\
\hline 10 & 15 \\
\hline 20 & 10
\end{tabular}

e o conjunto de alguns de pontos para a restrição 2:

\begin{tabular}{c|c}
$x_{1}$ & $x_{2}$ \\
\hline 0 & 60 \\
\hline 10 & 30 \\
\hline 20 & 0
\end{tabular}

O gráfico resultante é dado na Figura 1. Observamos que os pontos A e B representam a interseção da restrição 1 com o eixo y e da restrição 2 com o eixo $x$, respectivamente. Já o ponto $C$ representa e interseção entre as duas restrições.

Figure 1 - Gráfico das restrições 1 e 2.

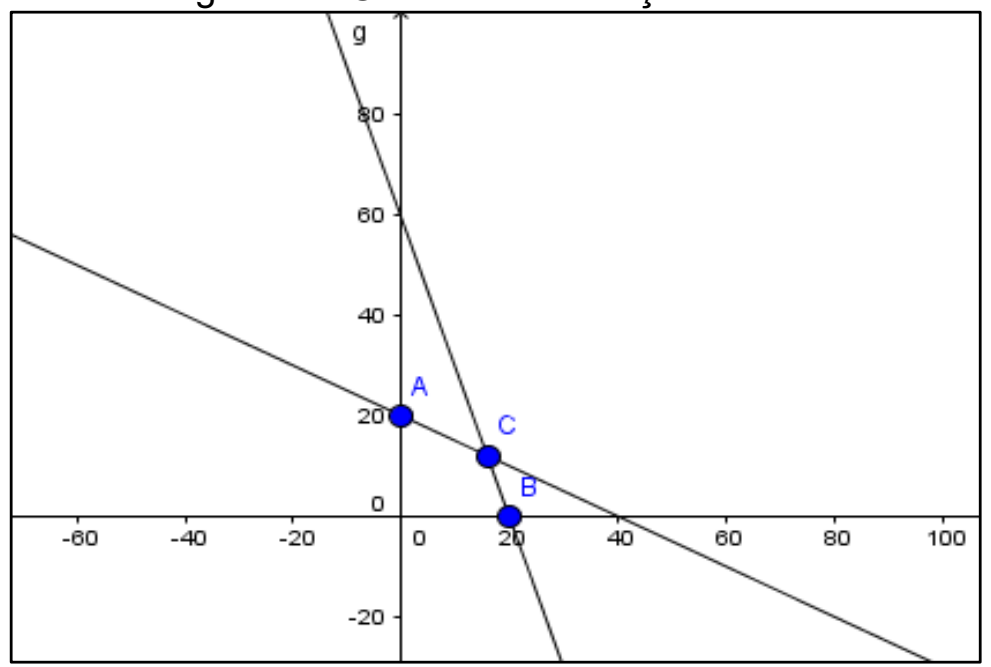

Fonte: Dados da pesquisa.

Acrescentando as chamadas variáveis de folga, denotadas por $s_{1}$ e $s_{2}$, iremos transformar as inequações em equações:

$$
\begin{gathered}
Z: 100 x_{1}+80 x_{2}=0 \\
2 x_{1}+4 x_{2}+s_{1}=80 \\
3 x_{1}+x_{2}+s_{2}=60
\end{gathered}
$$


Portanto, note que para $x_{1}$ valer zero o resultado a ser esperado para a expressão $2 x_{1}+4 x_{2}$ deve totalizar 80. Caso seja maior que zero, a expressão $2 x_{1}+4 x_{2}$ deve ser menor que 80 , o que efetivamente caracteriza a restrição.

Para obtermos o lucro máximo os alunos fizeram uma investigação nos pontos do gráfico cartesiano e concluíram que os pontos $\boldsymbol{A}, \boldsymbol{B}$ e $\boldsymbol{C}$ são os mais importantes. A partir da verificação de cada ponto na equação $Z: 100 x_{1}+80 x_{2}$, chegaram aos seguintes resultados:

$$
\begin{gathered}
\boldsymbol{A}(\mathbf{0}, \mathbf{2 0}): 100 \times 0+80 \times 20=1600 \\
\boldsymbol{B}(\mathbf{2 0}, \mathbf{0}): 100 \times 20+80 \times 0=2000 \\
\boldsymbol{C}(\mathbf{1 6}, \mathbf{1 2}): 100 \times 16+80 \times 12=1600+960=2560
\end{gathered}
$$

Portanto, a partir da comparação dos resultados, os alunos chegaram à conclusão de que o ponto $\boldsymbol{A}$ ilustra o maior lucro obtido nessa situação.

\section{c) Verificação da solução e formalização dos conceitos}

Por meio da verificação da solução e o uso da notação matemática, os alunos foram capazes de obter a solução esperada para o problema. Além disso, foram introduzidos nessa atividade os conceitos de equações lineares, gráficos de retas no plano cartesiano e interseção de retas.

O problema gerador 2 ilustra um exemplo de tomada de decisão sobre a oferta de emprego. O objetivo da atividade é reforçar os conceitos matemáticos desenvolvidos no problema gerador 1 . Com isso, buscamos dar sentido à Matemática vista em sala de aula com o cotidiano dos alunos.

ii) Problema 2 (Tomada de decisão - Oferta de emprego): Consiste em escolher qual a melhor opção entre 2 alternativas de ofertas de emprego:

1. 10 reais por hora trabalhada mais $2 \%$ de comissão sobre as vendas.

2. 8 reais por hora trabalhada mais $3 \%$ de comissão sobre as vendas.

\section{a) Estabelecendo um plano}

A incógnita envolvida é $x$, referente ao número de vendas. Denotamos por A como o valor recebido pela primeira oferta de emprego e $B$ o valor recebido na segunda oferta. $O$ equacionamento obtido é:

$$
\begin{gathered}
A: 10+2 \% x=10+0,02 x \\
B: 8+3 \% x=10+0,03 x
\end{gathered}
$$

\section{b) Execução do plano}

Observamos que as equações obtidas representam retas e, então, foram construídas as tabelas para a construção do gráfico. O eixo $x$ representa o número de vendas e o eixo $y$ representa o salário obtido para cada oferta de emprego. O gráfico resultante é dado na Figura 2. 


\begin{tabular}{c|c}
$x$ & $\boldsymbol{A}$ \\
\hline 0 & 10 \\
\hline 10 & 10,20 \\
\hline 20 & 10,40 \\
\hline 30 & 10,60
\end{tabular}

\begin{tabular}{c|c}
$x$ & $\boldsymbol{B}$ \\
\hline 10 & 8,30 \\
\hline 20 & 8,60 \\
\hline 30 & 8,90
\end{tabular}

Figure 2 - Gráfico resultante.

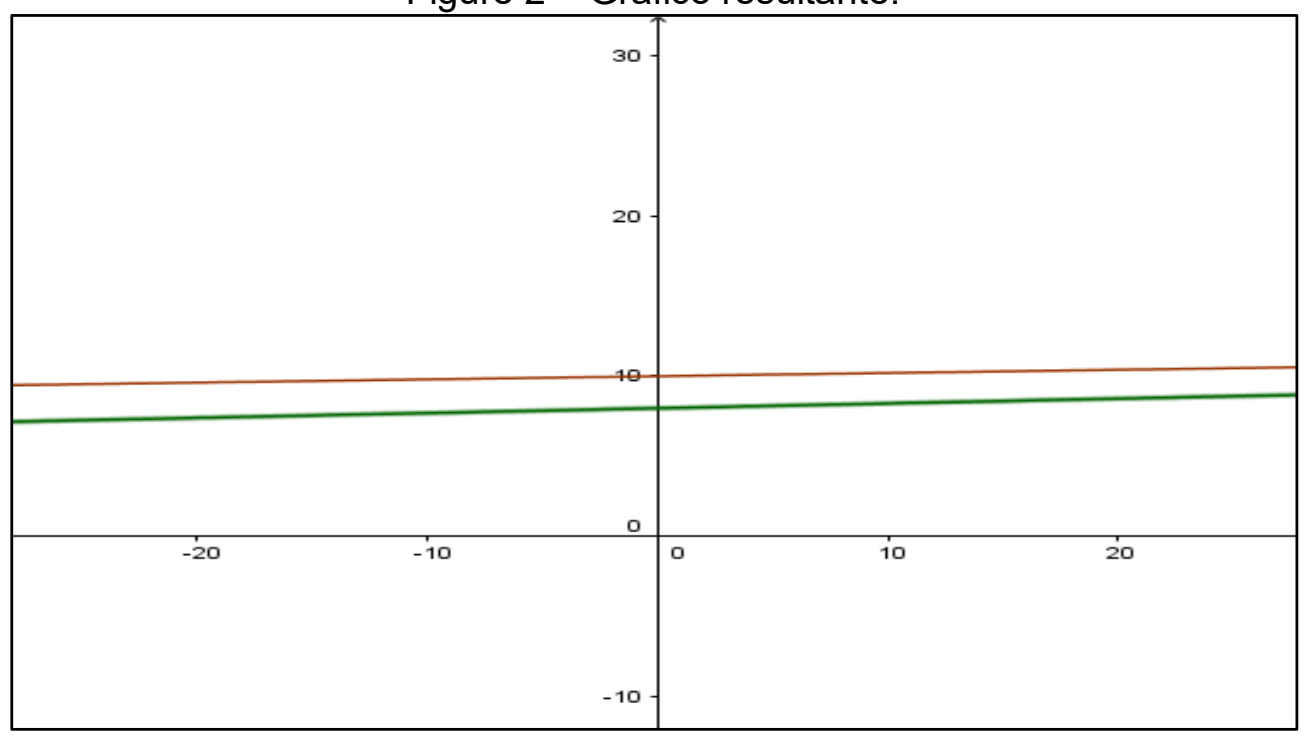

Fonte: Dados da pesquisa.

Por meio da construção do gráfico é possível observar que a solução é ilimitada, ou seja, quanto mais vende, mais ganha; portanto, até aqui a melhor opção é a $\boldsymbol{A}$. Durante a resolução surgiu o seguinte questionamento: Quanto devemos vender para ganharmos o mesmo salário em $A$ e $B$ ?

O mesmo salário acontece quando $\boldsymbol{A}=\boldsymbol{B}$, ou seja:

$$
\begin{gathered}
10+0,02 x=8+0,03 x \\
2=0,01 x \\
x=2 / 0,01=200
\end{gathered}
$$

Se vendermos $x=200$ produtos, vamos receber o mesmo salário. Comprovação:

$$
\begin{gathered}
A=10+0,02 \times 200=10+4=14 \\
B=8+0,03 \times 200=8+6=14
\end{gathered}
$$

\section{c) Verificação e consolidação dos conceitos matemáticos}

Por meio da resolução do problema gerador 2, os alunos foram capazes de construir as retas correspondentes a cada situação e verificar a relação de cada uma das variáveis. Os conceitos matemáticos introduzidos foram equações lineares, construção de gráficos e interseção de retas. 


\subsection{Comentários}

Vale destacar que, durante a aplicação das atividades, os problemas apresentados despertaram a curiosidade dos alunos, o querer obter a resposta, com a intervenção do professor, através de questões motivadoras, os alunos foram compreendendo o problema (primeira fase da resolução de problemas), buscando as incógnitas, os dados, as condições necessárias para a formulação do problema. Em um segundo momento, com a intervenção do professor, buscou-se estabelecer um plano. Os alunos apresentaram suas indagações, estratégias para resolver o problema, neste momento houve uma construção coletiva do plano a ser seguido após um momento de discussão. Com o plano estabelecido, os alunos passaram a executá-lo a fim de obter a solução do problema. Alguns alunos apresentaram dificuldades para executar o plano, mas, com a ajuda dos colegas, foi possível resolver o problema. Ao final das atividades o professor, juntamente com os alunos, realizaram a validação da solução.

Para finalizar a atividade didática, cada um dos alunos apresentou seu relato sobre as atividades realizadas. De forma geral, os alunos apresentaram relatos positivos, pois os problemas despertaram neles a curiosidade e a vontade de encontrar a solução e, para isso, os conceitos matemáticos envolvidos foram facilmente introduzidos. Vale destacar que, inicialmente, os alunos se mostraram apreensivos em relação à metodologia apresentada, pois esperavam que o professor transferisse todo o conhecimento, visto que é o modelo de ensino que eles têm contato atualmente. Da forma como ocorreu, o professor se mostra como mediador do conhecimento.

Assim, podemos concluir que o objetivo do projeto foi alcançado, uma vez que conseguimos despertar a curiosidade e o gosto pela matemática, levando os alunos à construção do próprio conhecimento matemático através da mediação do professor com questão estimulantes sobre os problemas abordados.

\section{Considerações finais}

Um dos grandes desafios na atualidade está em levar o conteúdo matemático para o aluno de forma aplicada a situações reais, que incentivem e facilitem sua compreensão. Desta forma, os problemas de otimização linear auxiliam na contextualização de problemas práticos, principalmente os vivenciados por pequenas indústrias, para o ensino de equações Lineares e estudo de retas. A metodologia de ensino empregada com base na Resolução de Problemas contribuiu para as aplicações destes problemas, de forma a tornar o ensino de matemática mais atrativo aos estudantes, tornando-os agentes de seu aprendizado.

A utilização da resolução de problemas como metodologia de ensino favoreceu uma boa interação, socialização e discussão das estratégias utilizadas pelos alunos (ONUCHIC, 2013). A forma como o conhecimento matemático é construído, a partir do uso da Resolução de problemas, apoiado na intuição e conhecimentos prévios dos alunos até se chegar a uma generalização, torna 
o ensino de matemática mais desafiador para professores, como mediadores, e para os alunos, como principais agentes nesse processo.

Desta forma, o presente trabalho apresenta implicações para a sala de aula no Ensino Médio e propõe o ensino de equações lineares e seus gráficos por meio de problemas de otimização linear usando como metodologia de ensino a Resolução de Problemas. As atividades desenvolvidas proporcionam ao aluno a relação do conteúdo matemático com a prática e com situações de tomada de decisões. Assim, esses estudos contribuem para a formação dos alunos enquanto profissionais e cidadãos, com conhecimento sólido de conceitos matemáticos, habilidade para decisões e aprendizagem significativa.

Portanto, os estudos aqui desenvolvidos desempenham um importante papel na formação dos alunos e contribuem para a conquista da cidadania além do desenvolvimento das capacidades relativas a aspectos cognitivos, afetivos e éticos. Esperamos que esta pesquisa venha a contribuir com futuras investigações no que se refere à aprendizagem de equações lineares e retas e que a proposta didática aqui apresentada sirva como inspiração aos professores deste nível de ensino, além de oferecer instrumentos para a compreensão e intervenção da realidade social.

\section{Referências}

AZEVEDO, C. G. da. Problemas de Otimização no Ensino Médio. 2015. 62 f. Dissertação (Mestrado em Matemática) - Programa de Mestrado Profissional em Matemática em Rede Nacional, Universidade Federal do Amazonas, Manaus, 2015.

FONSECA, M. C. F. R. O sentido matemático do letramento nas práticas sociais. Presença Pedagógica, Belo Horizonte, edição 64, jul./ago. 2005.

GOLDBARG, M. C.; LUNA, H. P. L. Otimização combinatória e programação linear. 2. ed. rev. Rio de Janeiro: Elsevier, 2005.

LACHTERMACHER G. Pesquisa Operacional na tomada de decisões. São paulo: Pearson Prentice Hall, 2009.

LEAL JUNIOR, L. C.; ONUCHIC, L. de la R. Ensino e Aprendizagem de Matemática Através da Resolução de Problemas Como Prática Sociointeracionista. Bolema, Rio Claro, SP, v. 29, n. 53, p. 955-978, 2015.

LINS, M. P. E.; CALOBA, G. M. Programação Linear: com aplicações em teoria dos jogos e avaliação do desempenho. Rio de Janeiro: Interciência, 2006.

ONUCHIC, L. de la R; ALLEVATO, N. S. G.; NOGUTI, F. C. H.; JUSTULIN, A. M. (Orgs.). Resolução de Problemas: Teoria e Prática. 1. ed. Jundiaí, SP: Paco Editorial, 2014.

ONUCHIC, L. de la R. A resolução de problemas na Educação Matemática: onde estamos? E para onde iremos? Espaço Pedagógico. v. 20, n. 1, p. 88-104, 2013.

ONUCHIC, L. de la R. Ensino-aprendizagem de Matemática Através da Resolução de Problemas. In: BICUDO, M. A. V. Pesquisa em Educação Matemática: concepções e perspectivas. São Paulo: UNESP, 1999. 
POLYA, G. A Arte de Resolver Problemas. 2. ed. Rio de Janeiro: Interciência, 2006.

ROCHA, A. M. Problemas de otimização envolvendo a matemática do Ensino Médio. 2013. 52 f. Dissertação (Mestrado em Matemática) - Programa de Mestrado Profissional em Matemática em Rede Nacional, Instituto de Matemática e Estatística, Universidade Federal de Goiás, 2013.

SANTOS, V. O.; ALMEIDA, V. L. Matemágica e Resolução de Problemas. REMAT: Revista Eletrônica da Matemática, Bento Gonçalves, v. 4, n. 1, p. 147-162, ago. 2018.

SILVA, D. P. da; GUERRA, E. A. A aprendizagem de Análise Combinatória no Ensino Médio: uma proposta didática por meio da Resolução de Problemas. REMAT: Revista Eletrônica da Matemática, Bento Gonçalves, v. 3, n. 2, p. 40-51, dez. 2017. 